\title{
Reconstructing the Acoustic Pressure Field Based on Oblate Spheroidal Coordinates
}

\author{
Yang Ruiliang \\ School of Mechanical Engineering \\ Tianjin Polytechnic University \\ Tianjin, China \\ Yangruiliang2001@sina.com
}

\author{
Zhang Xiaojing \\ University for Science \& Technology Zhengzhou \\ Zhengzhou, China \\ 43188940@qq.com
}

\begin{abstract}
A new acoustic reconstructing method based on oblate spheroidal coordinates is proposed and deduced in this paper. The acoustic pressures of whole acoustic field are expressed by the product of the coefficients and the conventional interpolate polynomial. Once the coefficients are specified by the pressures of the measured points, the least-squares method and the singular value decomposition (SVD) method, the whole acoustic field can be reconstructed. The traditional HELS method is most suitable for reconstructing pressures radiated a spherical source or sources with a characteristic aspect ratio $x: y: z$ close to $1: 1: 1$. While the new method proposed in this paper is suitable for the acoustic sources with a characteristic aspect ratio $y: z$ close to $1: 1$ and shorter $z$, which extends the appropriate application domain of traditional HELS largely. The reconstructing examples of a dilating sphere and an oblate spheroid source show that the new method is feasible and efficient.
\end{abstract}

Keywords- acoustic reconstructing method; Helmholtz equation-least-squares (HELS) method; oblate spheroidal coordinates; acoustic pressure field;

\section{INTRODUCTION}

In order to provide understanding of medical imaging such as source locations, reconstructing the acoustic pressure field is important and has been the subject of extensive studies for the past few decades. In order to deal with this kind of acoustical inverse problem, many techniques have evolved: Near-field acoustical holography (NAH) [1], the boundary element method (BEM) [2], Helmholtz equation-least-squares (HELS) method [3] etc. $\mathrm{NAH}$ is a well-known technique for the reconstruction acoustic pressure field. In its usual form, the principle consists in measuring sound pressure on a microphone grid on a given plane, calculating the pressure field in the kspace by a $2 \mathrm{D}$ Fourier transform and computing, ,acoustic back-propagation eee by using the inverse solution of the wave equation in the normal direction of the plane [4]. However, the reconstructing noises such as braking and riveting are often encountered in practice [5]. The BEM is original methods and remain the method of choice by many researchers and code developers. However, using the traditional boundary element method (BEM) to solve acoustic inverse problems requires too much time and known information [6]. In 1997, the HELS method was developed by Wang et al [3]. The HELS method has shown a great promise in reconstructing acoustic fields in both exterior [3] and interior acoustic regions [7], and has been successfully used to reconstruct acoustic radiation from a simplified engine block [8] and a ve-hicle front end [9]. However, the HELS method is based on the spherical wave expansion, thus the HELS method is most suitable for reconstructing pressures radiated a spherical source or sources with a characteristic aspect ratio $\mathrm{x}$ : $\mathrm{y}$ : $\mathrm{z}$ close to $1: 1: 1$.

In fact, there are a lot of sound sources with irregular shape. Thus, further extending the appropriate application domain of traditional HELS is necessary. This paper proposes a new acoustic reconstructing method, which is based on oblate spheroidal coordinates.

\section{PRINCIPLE}

\section{A. Oblate spheroidal coordinates}

Here introduces the oblate spheroidal coordinates $(r, \theta, \varphi)$, which are defined by

$$
\left\{\begin{array}{c}
x=r \sin \theta \cos \varphi \\
y=r \sin \theta \sin \varphi \\
z=\left(r^{2}-f^{2}\right)^{1 / 2} \cos (\theta)
\end{array}\right.
$$

Where $(x, y, z)$ are the traditional Cartesian coordinates.

Let $r=r_{1}$, yielding the surface:

$$
\frac{x^{2}+y^{2}}{r_{1}^{2}}+\frac{z^{2}}{r_{1}^{2}-f^{2}}=1
$$

In Cartesian coordinates, which is the surface obtained by rotating an ellipse with semi-major axis $r_{1}$ and foci at $(0,0, \pm f)$ about its minor axis. The surface of $\theta=\mathrm{const}$ is one half of a one-sheeted hyperboloid. The surface of $\phi=$ const is a plane containing the $z$ axis. These surfaces are mutually orthogonal.

When $f=0$, the oblate spheroid reduces to sphere, then Eq.(2) can transform to usual spherical coordinates.

\section{B. Reconstructing the acoustic pressure field}

Consider an arbitrary structure immersed in an infinite homogeneous fluid and vibrating at constant 
frequency, $e^{i k r}$. The acoustic pressure values are known at certain points in the acoustic field:

$$
P\left(x_{i}\right)=P_{O i} \quad(i=1,2 \ldots M)
$$

Then the acoustic pressure field can be reconstructed as follows.

An artificial oblate spheroid with $r=r_{1}$ that surrounds the structure is drawn. The region of the structure and the artificial boundary is divided by $N$ domains ( $M \geq N$ ), with each domains as far as possible including one known point to guarantee the accuracy of reconstructing result.

The acoustic pressure at point $x(r, \theta, \varphi)$ can be expressed as:

$$
P(x)=\sum_{j=1}^{N} C_{j} \times \psi_{j}
$$

Where $N$ denotes the number of the nodes in the acoustic field, $C_{j}$ denotes the coefficient, the term $\psi_{j}$ is interpolate polynomial, which can be defined as [10][11]:

$$
\psi_{j}=e^{-i k\left(r-r_{1}\right)} \times\left(r_{1} / r\right) \times F_{j}\left(r_{1} / r\right) \times \psi_{j}^{(\varphi)} \times \psi_{j}^{(\theta)}
$$

The term $F_{j}\left(r_{1} / r\right)$ is a polynomial order $(n-1)$ and can be chosen in a variety of ways, which include the Lagrange interpolation polynomial, the shifted Legendre polynomial and the shifted Chebyshev one. $\psi_{j}^{(\varphi)}, \psi_{j}^{(\theta)}$ are "angular" functions that interpolate along oblate spheroidal surfaces, respectively. Here the "angular" shape functions are chosen as the conventional 1-D polynomials.

Substituting Eq.(4) into Eq.(3) yields:

$$
\left[\begin{array}{rrrr}
\psi_{11} & \psi_{12} & \mathrm{~L} & \psi_{1 N} \\
\psi_{21} & \psi_{22} & \mathrm{~L} & \psi_{2 N} \\
\mathrm{M} & \mathrm{M} & \mathrm{O} & \mathrm{M} \\
\psi_{M 1} & \psi_{M 2} & \mathrm{~L} & \psi_{M N}
\end{array}\right]\left\{\begin{array}{c}
C_{1} \\
C_{2} \\
\mathrm{M} \\
C_{N}
\end{array}\right\}=\left\{\begin{array}{c}
P_{O 1} \\
P_{O 2} \\
\mathrm{M} \\
P_{O M}
\end{array}\right\}
$$

Let

$$
I=\sum_{m=1}^{M}\left(\sum_{n=1}^{N} C_{n} \psi_{m n}-P_{0 m}\right)^{2}
$$

The errors involved in the approximate solution can be minimized by the least-squares method:

$$
\frac{\partial I}{\partial C_{n}}=0
$$

Substituting Eq.(7) into Eq.(8) and using the SVD method yield:

$$
\{C\}=\left(\left[\psi_{M N}\right]^{T}\left[\psi_{M N}\right]\right)^{-1}\left[\psi_{M N}\right]^{T}\{\xi\}
$$

Where the element of the norm $\{\xi\}$ is given by $\xi_{i}=P_{0 i} \psi_{i}$. And $\left(\left[\psi_{M N}\right]^{T}\left[\psi_{M N}\right]\right)^{-1}\left[\psi_{M N}\right]^{T}$ is the pseudoinverse of $\psi_{M N}$ using the SVD method. The superscript $T$ and -1 stands for a transposition and the inverse of the matrix. Then the acoustic pressure anywhere in the acoustic field can be obtained

$$
\begin{aligned}
& P(x)=\left\{\psi_{1}(x) \quad \psi_{2}(x) \quad \mathrm{L} \quad \psi_{N}(x)\right\} \\
& \left(\left[\psi_{M N}\right]^{T}\left[\psi_{M N}\right]\right)^{-1}\left[\psi_{M N}\right]^{T}\left\{\begin{array}{c}
P_{O 1} \\
P_{O 2} \\
\mathrm{M} \\
P_{O M}
\end{array}\right\}
\end{aligned}
$$

When acoustic pressures of known points are obtained, the pressure everywhere in the acoustic field can be approximated using Eq. (10).

\section{EXAMPLES}

Two examples are included in this part: (1) a dilating sphere, (2) a source with the shape of an oblate spheroid.

\section{A. A dilating sphere}

A dilating sphere with radius of $a$ is immersed in the air. The acoustic pressures of four points on a hypothetical concentric spherical surface at $r=2 a$, $\theta=0$ and $\phi$ varying from 0 to $\pi$ at a uniform interval are known. Then the acoustic pressure of the whole acoustic field can be reconstructed.

Angular shape functions and radial shape functions are all chosen as the Lagrange interpolation polynomial. Fig .1 gives the normalized real and imaginary pressure on the surface of a dilating sphere as a function of nondimensional wavenumber, where $k$ is wavenumber. For the range of $k a$ "s presented, the pressures computed with the novel method for all practical purposes agreed exactly with the theory.

\section{B. A source with the shape of an oblate spheroid}

A source with the shape of an oblate spheroid with $r_{1}=1$ and $f=0.2$ is immersed in air of density 1.225 $\mathrm{kg} / \mathrm{m}^{3}$ and acoustic speed of $340 \mathrm{~m} / \mathrm{s}$ (see Fig .2). Forty known points are uniformly distributed in an oblate spheroid with $r_{1}=2$ and $f=0.2$. The acoustic pressures of forty known points are knows, then the acoustic pressure of the whole acoustic field can be reconstructed.

For the convenience, the source is assumed to dilate uniformly in all directions and the frequency is $54.133 \mathrm{HZ}$. And then the acoustic pressures of known points can be derived by using the BEM method. Since the acoustic 
pressures of knows points are obtained, the reconstructing process can be done.

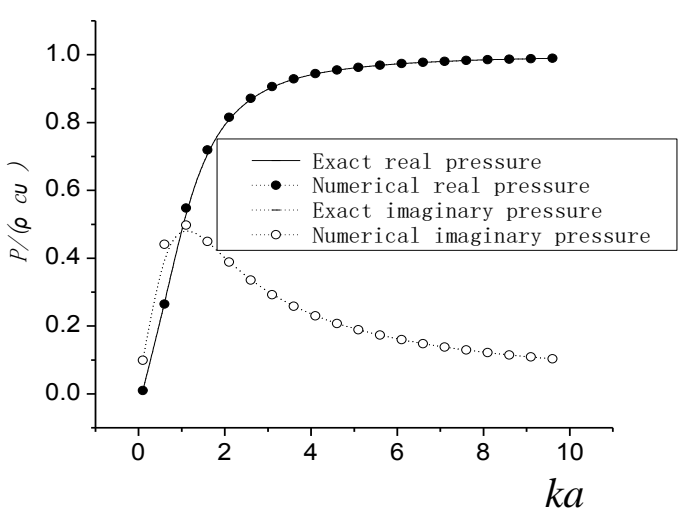

Figure 1. Variation of normalized surface pressure with nondimensional wavenumber $k a$ for a dilating sphere

TABLE I shows the comprasion of the calculationed pressuress and the exact pressures. It can be seen from the table 1 that the reconstructing pressures are close to the exact pressures.

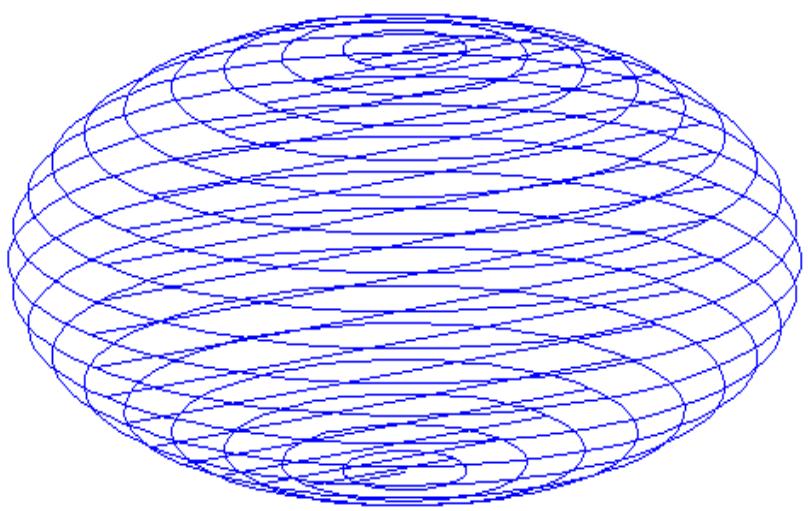

Figure 2. Illustration of with the shape of an oblate spheroid

TABLEI. THE COMPARISON OF THE RECONSTRUCTING SURFACE PRESSURES AND THE EXACT PRESSURES

\begin{tabular}{|c|c|c|}
\hline$\varphi(0)$ & $\begin{array}{c}\text { Exact } \\
\text { surface } \\
\text { pressures }\end{array}$ & $\begin{array}{c}\text { The } \\
\text { reconstructing } \\
\text { surface pressures }\end{array}$ \\
\hline 0.0000 & 1.0000 & 1.0184 \\
\hline 15.6641 & 1.0000 & 0.9926 \\
\hline 28.3778 & 1.0000 & 0.9823 \\
\hline 40.0252 & 1.0000 & 0.9914 \\
\hline 50.7251 & 1.0000 & 0.9863 \\
\hline 60.7377 & 1.0000 & 0.9702 \\
\hline 70.1050 & 1.0000 & 0.9886 \\
\hline 79.5656 & 1.0000 & 0.9682 \\
\hline 90.0000 & 1.0000 & 0.9961 \\
\hline
\end{tabular}

\section{DISCUSSION}

The HELS method is based on the spherical wave expansion, thus the HELS method is most suitable for reconstructing pressures radiated a spherical source or sources with a characteristic aspect ratio $\mathrm{x}: \mathrm{y}: \mathrm{z}$ close to $1: 1: 1$, but is not suitable for reconstructing pressures from a source similar to oblate spheroidal coordinates. While the method proposed in this paper is suit for a source with a character aspect ratio y: $\mathrm{z}$ close $1: 1$ and shorter $\mathrm{z}$.

In fact, there are a lot of sound sources with irregular shape. Thus, further extending the appropriate application domain of traditional HELS is necessary.

To compare the application domain of the two methods, an oblate spheroid was chose as follows. The major and minor axes of the ellipsoid are 1 meter and 0.5 meter, respectively. The frequency is $600 \mathrm{HZ}$. The oblate spheroid is immersed in air of density $1.225 \mathrm{~kg} / \mathrm{m}^{3}$ and acoustic speed of $340 \mathrm{~m} / \mathrm{s}$. Twenty measured points are uniformity distributed in ellipses with a 2 meters radius and $\theta=0$. Others twenty measured points are uniformity distributed in ellipses with a 3 meters radius and $\theta=0$. There are 40 measured points in all

To make the result comparable, we suppose that the oblate spheroid surface is uniformity distributed with unit pressure. Then the pressure everywhere, including the forty measured points and the surface, can be obtained using the BEM.

The surface pressures can be obtained by means of this paper with respect to measured point's pressures. Fig .3 shows the comprasion of the calculationed pressure and the exact pressure, where the calculationed pressure include the pressure using the BEM, the reconstructing pressure using this paper, the reconstructing pressure using the HELS methods with 4 terms, the reconstructing pressure using the HELS methods with 9 terms. It can be seen from Fig .3 that the calculated errors using the novel method in this paper is relatively low at the high frequency $(k a=11.088)$. The calculated time using the novel method in this paper is about 0.04 second, while the calculated time using the HELS method with 4 terms is more than 1.5 second( not including the time of orthonormalization, furthermore, the calculated time increases as the terms increase using the HELS method). Thus using the traditional HELS method, not only the calculated time is much more than that using the novel method, but also the calculated errors are much more than that using the novel method.

Thus, in comparison to the traditional HELS method, the efficiency and precision of reconstruction can be significantly enhanced and satisfactory reconstruction can be obtained with relatively few measurements using the new method, especially when reconstructing the source of an oblate spheroidal. Numerical example of reconstruction of acoustic radiation from two shapes of the sound source is successful. 


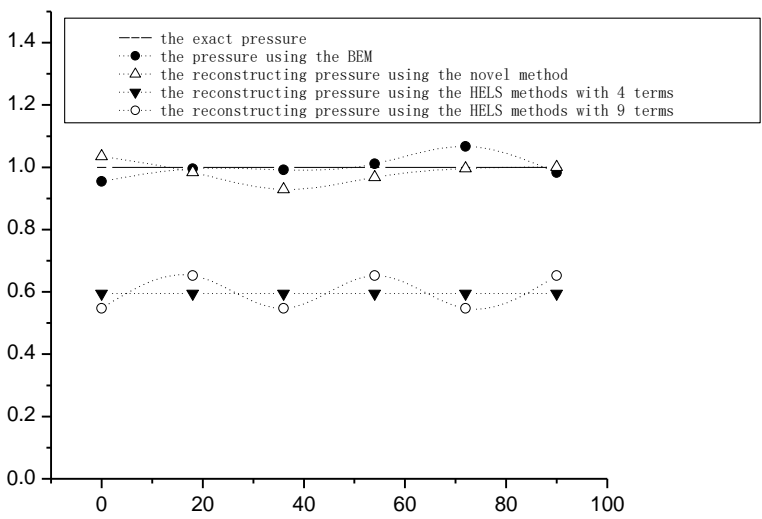

Figure 3. The comparison of the calculationed pressure and the exact pressure

\section{CONCLUSIONS}

The HELS method is based on the spherical wave expansion, thus the suitable range of the HELS method is most suitable for reconstructing pressures radiated a spherical source or sources with a characteristic aspect ratio $\mathrm{x}$ : $\mathrm{y}$ : $\mathrm{z}$ close 1:1:1 [3]. The literature [10] used spheroidal wave functions instead of the spherical wave functions, which extends the appropriate application domain of traditional HELS to the source similar to spheroidal surface. But the precision and the efficiency of that method is unsatisfactory. The literature [11] reconstructs the acoustic pressure field based on ellipsoidal coordinates, which extends the appropriate application domain of traditional HELS to the source with arbitrary shape. But the ellipsoidal coordinates are very complicated, which results in the increase of computational difficulty and the decline of computational efficiency.

On the basis of previous research [10] [11], this paper proposes a new acoustic reconstructing method based on the oblate spheroidal coordinates, thus the new method is most suitable for reconstructing the acoustic field radiated not only from a spherical source, but also from a source with a character aspect ratio $y: z$ close $1: 1$ and shorter $z$. In comparison to the traditional HELS method, the efficiency and precision of reconstruction can be significantly enhanced and satisfactory reconstruction can be obtained with relatively few measurements using the new method, especially when reconstructing the source of an oblate spheroidal. Numerical example of reconstruction of acoustic radiation from two shapes of the sound source is successful.

\section{ACKNOWLEDGMENT}

This research was supported by the NSFC (the Natural Science Foundation of China) No. 51208527 and Funding Scheme for Young Teachers of Higher School in Henan Province (2012GGJS -124).

\section{REFERENCES}

[1] J.H. Thomas, V.Grulier, S.Paillasseur, J.C.Pascal, J.C.LeRoux, "Real-time near-field acoustic holography for continuously visualizing nonstationary acoustic fields", Journal of the Acoustica Society of America, 2010, 128:, 3554-3567.

[2] G.T. Kim, B. T. Lee, "3D sound source reconstruction and field reproduction using the Helmholtz integral equation", Journal of Sound and Vibration, 1990, 136: 245-261.

[3] W. Zhaoxi, W. Sean, "Helmholtz. equation-least-squares method for reconstructing the acoustic pressure field", Journal of the Acoustical Society of America, 1997, 102: 2020-2032.

[4] C. Pezerat, Q.Leclere, N.Totaro, M.Pachebat, "Identification of vibration excitations from acoustic measurements using near field acoustic holography and the force analysis technique", Journal of Sound and Vibration, 2009, 326: 540-556.

[5] P. Siwei, J. Weikang, "A hybrid approach to reconstruct transient sound field based on the free-field time reversal method and interpolated time-domain equivalent source method", Journal of Sound and Vibration, 2014, 333: 3625-3638.

[6] Z. Zhang, N. Vlahopoulos, S.T. Raveendra, T. Allen, K.Y. Zhang "A computational acoustic field reconstruction process based on an indirect boundary element formulation", Journal of the Acoustical Society of America, 2000, 108: 2167-2178.

[7] Rayess N, W. Sean. "Experimental validations of the HELS method for reconstructing acoustic radiation from a complex vibrating structure". Journal of the Acoustical Society of America, 2000,107: 2955-2964.

[8] S. F. Wu, N. Rayess. "Visualizing sound radiation from a vehicle front end using the HELS method", Journal of Sound and Vibration, 2001, 248: 963 - 974.

[9] Wu SF, Zhao X. "Combined Helmholtz equation-least squares method for reconstructing acoustic radiation from arbitrarily shaped objects", Journal of the Acoustical Society of America, 2002, 112: 179-188.

[10] Z. Yide, W. Jianbo, Yang Ruiliang, F Xiaowei, "Reconstructing the acoustic pressure field using spheroidal wave functions", The XI th International Seminar/Workshop on Direct and Inverse Problems, 2006, 25-30.

[11] Y. Ruiliang, G. Long, "Reconstructing the acoustic pressure field based on ellipsoidal coordinate", The XIII th International Seminar/Workshop on Direct and Inverse Problems, 2008, 20-24. 